\title{
BILABIAL AND LABIO-DENTAL FRICATIVES IN EWE
}

\author{
Ian Maddieson
}

\section{Introduction*}

A voiceless labio-dental fricative, /f/, occurs in the phonological inventory of many (roughly 40\%) of the world's languages. A voiceless bilabial fricative, $/ \phi /$, occurs in considerably fewer-in only about $7 \%$ (Maddieson 1984). Although close to half of the languages have one or the other of these two segments, the number of languages that have a contrast between them is quite limited. A contrast between the voiced counterparts of these segments, namely $/ v, \beta /$, is also relatively unusual. To most phoneticians the acoustic difference between these pairs is quite subtle and some expect such subtle contrasts to be avoided. However, based on the separate frequencies of /f/ and $/ \phi /$ only a small proportion of languages (about 3\%) would be expected to have both if their occurrences were quite independent of each other. It is therefore not clear that a contrast between bilabial and labio-dental fricatives is actually avoided in the consonant inventories of languages. In any case, it is interesting to examine the production of these segments in a language with both, as this may be generally instructive about how phonetic contrasts of a more subtle than average degree are realized. Moreover, although the movements of the lips have been quite widely studied in the production of labial stops and nasals (e.g. Fujimura 1961, Lubker \& Parris 1969, Sussman et al 1973, Dunn 1993, Smith 1995, Löfqvist \& Gracco 2002, Löfqvist 2005), there is much less data on their

\footnotetext{
* The occasion of a joint field trip to Ghana in 1994 with Russ Schuh enabled further visual data to be collected on the topic of this paper. I would like to thank Russ for the cameraderie that we enjoyed on that trip, as well during the countless miles we covered together on Stone Canyon, the OJ run, in Franklin Canyon, on Casiano, Linda Flora, and many other spots. The articulographic data discussed in this paper were collected at MIT under a protocol approved by the MIT IRB. The assistance of Joseph Perkell, Melanie Mathies, and Joseph Svirsky is gratefully acknowledged. This research was supported in part by an NSF grant.
} 
action in fricatives. West Africa is one area of the world where a contrast between bilabial and labio-dental fricatives occurs and the production of both by the same individuals can therefore be studied. The contrast is particularly well-known from Ewe and some neighboring languages such as Logba (Ladefoged 1964) and Avatime (Schuh 1995), which may have been influenced by Ewe. Issues of interest include how different the movements of both the upper and lower lips are in the two kinds of labial fricatives, whether the two lips interact with each other, if the required positions for the production of these fricatives are achieved by essentially independent movement of the lips or include a major component of activity by the jaw to raise the lower lip, and whether there is any influence of voicing on the articulations.

A further question concerns whether evidence of phonetic "enhancement" can be found in the production of the bilabial/labio-dental fricative contrast. Enhancement theories are of two general types. In one (e.g., Stevens \& Keyser 1989), subsidiary phonetic properties are seen as normally co-occurring with primary properties to enhance the effectiveness of those primary properties, as in the occurrence of rounding with non-low back vowels. In the other (e.g., Keating 1984, Padgett \& Zygis 2003), enhancement is seen as dependent on the phonological system of a given language, as suggested for voicing categories in obstruents. The occurrence of enhancement in accord with both these types of ideas has been held to occur in the production of labial fricatives. In an observation first published in the Phonetic Study of West African Languages (1964), Ladefoged suggested that languages with both labio-dental and bilabial fricatives in their consonant inventory display a different articulation for their labio-dentals from that found in a language which does not contrast them with bilabials. Ladefoged (1990, see also Ladefoged 1993) later elaborated on this point based on video of the well-known Ghanaian linguist Gilbert Ansre, a speaker of the Ewe language as spoken in parts of the Volta Region of Ghana around Kpando. The specific proposal is that the upper lip is actively raised in production of labio-dentals in languages with this contrast. This idea thus falls within the family of proposals suggesting that the structure of a system of phonological contrasts permits some predictions to be made about the articulatory or acoustic realizations of the segments it contains, and in particular that some of these predictions can be explained by the hypothesis that speakers make efforts to enhance the perceptibility of contrasts. An active gesture of upper lip raising would certainly contribute to making the visual recognition of the segmental distinction in question clearer - and the important role played by visual cues in the perception of language has been more clearly recognized in recent years (see, e.g. Massaro 1997). It is less 
clear that such a gesture would effectively enhance the acoustic difference between the segments. The other type of enhancement is suggested by Pulleyblank (2003: 731) who asserts that "labiodental fricatives typically involve some protrusion of the lower lip" and that "languages like Ewe...oppose [-round] bilabials to [+round] labiodentals." These ideas can be examined with precise data on the production of bilabial and labio-dental fricatives.

\section{Methods and Data}

The materials analyzed in this report are electromagnetic articulography records from two speakers, designated $\mathrm{M}$ and V, of the Kpando dialect of Ewe. These data were obtained using the MIT EMMA system described by Perkell et al. (1992). Electromagnetic articulography enables the position of specific points on a number of articulators to be tracked over time. Small receiver coils are attached to the articulators of interest using dental adhesive. These receiver coils receive signals generated by three transmitter coils producing alternating fields of magnetic flux at different frequencies. The transmitters are located near the chin, the crown of the head, and the nape of the neck. The relative strength of each different frequency signal at a receiver coil is proportional to the distance of the receiver from the transmitter coil. By triangulation the position of the coil can thus be determined. In this experiment, five receivers were placed on moveable articulators - two on the lips, on the outer surfaces of the upper and lower lip; two on the tongue, one on the front toward the back of the blade and one further back below the velum; and one attached at the base of the lower incisors to indicate movements of the jaw. Two further receivers were placed on the bridge of the nose and the upper incisors to provide fixed reference points on the skull to which the movements of articulators can be related. Although the speaker's head is stabilized by the helmetlike device on which the transmitters are mounted, these fixed reference points enable a correction to be applied in the case of unexpected movement of the head within the flux field. The position of each coil is sampled at a frequency of 312.5 $\mathrm{Hz}$, with location values reported in a two-dimensional sagittal plane with horizontal ( $\mathrm{x}$ ) and vertical $(\mathrm{y})$ vectors. At the same time a digital audio signal is recorded at $10 \mathrm{kHz}$. Since the EMMA system is quiet the quality of the audio recording is good. For the articulatory signals measurement precision is estimated to be at least on the order of $0.5 \mathrm{~mm}$. The movement signals are quantized in 0.1 mm steps.

Each speaker was prompted from written cues, primarily showing target words in short carrier phrases. The target words were grouped in two sets, the first 
designed to investigate labial-velar and simple bilabial and velar stops, and the second to investigate labial fricatives. Within each set the order of repetitions was randomized. The word-list for fricatives included the following quadruplet contrasting voiced and voiceless bilabial and labio-dental fricatives between /e/ vowels:

$\begin{array}{ll}{[\text { efe] }} & \text { efe 'nail; debt' } \\ \text { [eve] } & \text { eve 'two' } \\ {[\mathrm{e} \phi \mathrm{e}]} & \text { efe 'year' } \\ {[\mathrm{e} \beta \mathrm{e}]} & \text { eve 'Ewe people' }\end{array}$

These words were pronounced in the carrier phrase [ do be ni mama ni se] 'Say __ for grandma to hear'. Data from the pronunciation of these words will be the focus of this report. The intent was to obtain at least 10 repetitions of these and the other words on the list. Because of occasional misreadings, technical problems with the equipment, and data-handling errors, the number of usable repetitions per speaker actually obtained varies between 9 and 12. Using a display of the audio recording obtained simultaneously with the articulatory data, the midpoint of each fricative's acoustic duration was marked (this point being more readily identified than a precise onset or offset time) and an $800 \mathrm{~ms}$ long window of data beginning $500 \mathrm{~ms}$ before the marked point was downloaded to a separate file for each token. The repetitions of a given word can thus be examined in close time-alignment and average contours for the articulatory movements in the two planes can be calculated.

In addition to the articulographic data, video recordings of lip position have also been consulted. Two sources of this material were available. The first is the recording of Gilbert Ansre used in the preparation of Ladefoged (1990), and generously made available by Peter Ladefoged. The second are video recordings of seventeen speakers that I made in Accra in 1994. In the first video a mirror is placed to the side of the lips and thus simultaneous frontal and lateral views of the lip position are captured. In the second set of video data, separate recordings were made of frontal and lateral views of the lips.

Some of this data has been described in two conference papers (Maddieson 1993, 1995), but none of the articulographic results from the second speaker have previously been published. One reason for this is that the original post-processing of the data from this speaker seems not to have oriented the vertical and horizontal vectors correctly. Based on an examination of two palate traces, obtained at the end of the recording session, it was estimated that it was appropriate to rotate the 
axes $20^{\circ}$ clockwise. The palate traces are made by running one of the receiver coils, attached to the tip of one of the experimenter's fingers, over the speaker's hard palate. This procedure registers the contour of the palate in the same coordinate space as that in which the articulator positions are shown. The rotation orients the back of the hard palate horizontally, which is its typical orientation in a subject seated upright. This correction has been applied to all results reported below. In the uncorrected data the palate appeared to be sloping steeply upwards, and a falsely exaggerated displacement of the articulators in the horizontal plane was registered.

\section{EMMA Results}

The averaged movement trajectories of the three coils on the upper lip, lower lip, and lower incisor for the four words cited above are given in figures 1 and 2 for Speakers $\mathrm{V}$ and $\mathrm{M}$ respectively. The speaker should be pictured as facing to the left. Each panel in these figures represents the mean of the $\mathrm{X}$ and $\mathrm{Y}$ values of these coils over the 9-12 individual repetitions of the word shown, time-aligned at the center of the acoustic duration of the fricative. Labio-dental fricatives are shown in the left-hand panels of these figures, bilabials on the right. Voiceless fricatives are in the upper panels, voiced ones in the lower panels. For Speaker V, the movement shown covers the time interval from 150 to $750 \mathrm{~ms}$ of the 800 window for each token, and for Speaker M, who speaks a little more rapidly, the interval from 200 $\mathrm{ms}$ to $750 \mathrm{~ms}$. These points correspond well to the onset and end of the lip movements for production of the consonants in question. Distances on the horizontal and vertical axes are shown in $\mathrm{cm}$ on the same scale from an origin at the midpoint of the space enclosed by the transmitter coils. Arrows have been added to help clarify the path of movement of the lips in those words which have substantial movements that do not follow the same trajectory during their upward and downward excursions.

The overall patterns of movement have many similarities for the two speakers despite a certain number of differences. The main trends are the following: The upper lip makes minimal movements in labio-dental fricatives, but shows a consistent downward excursion in bilabials. The lower lip makes a substantial upward movement for all of the fricatives, but the displacement is greater for the labio-dentals than for the bilabials. The jaw makes only a small contribution to the upward movements of the lower lip. Voicing status has little impact on movement patterns - the voiceless and voiced counterparts are quite similar to each other. 

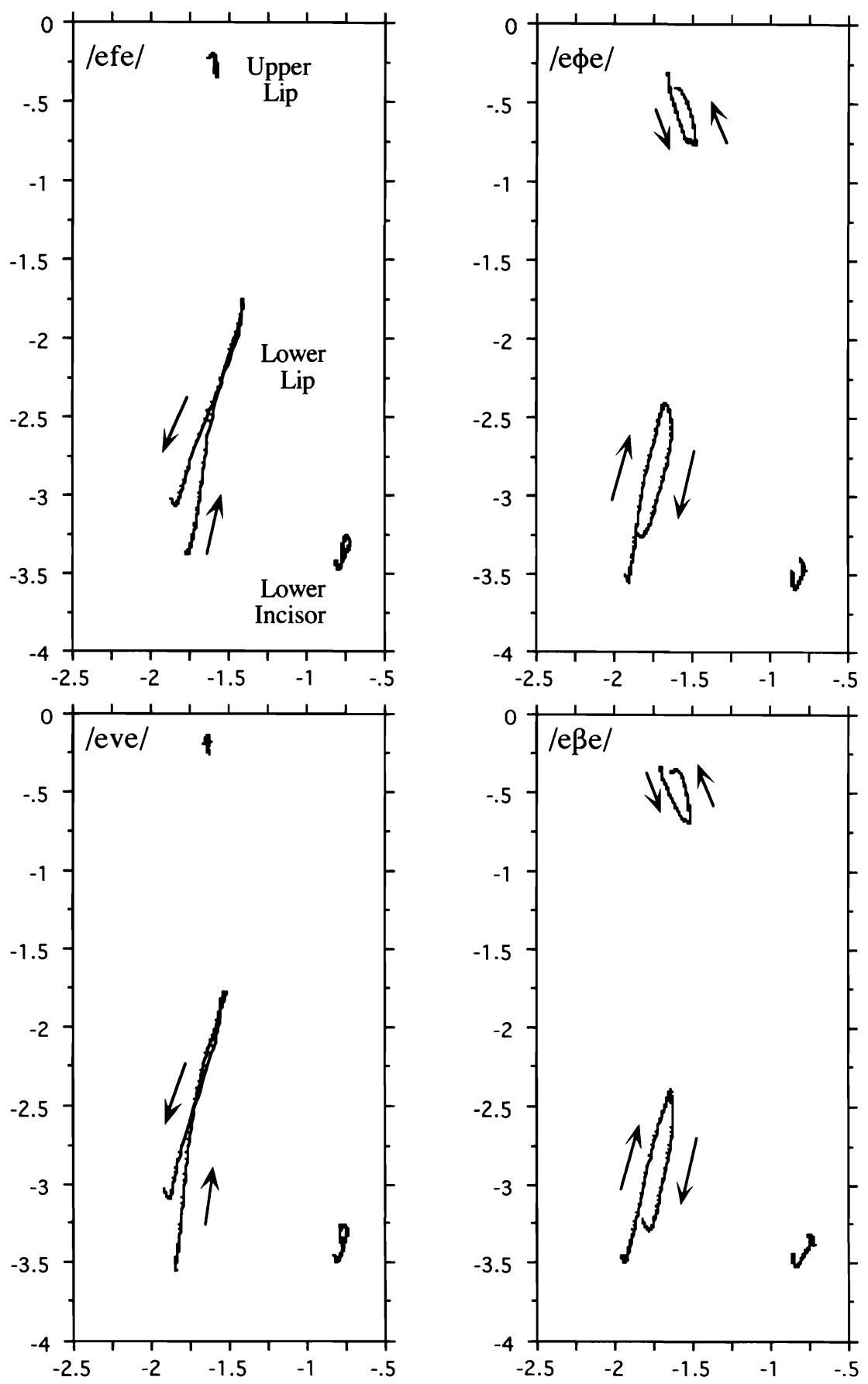

Figure 1. Lip and jaw movement trajectories for Speaker V. 

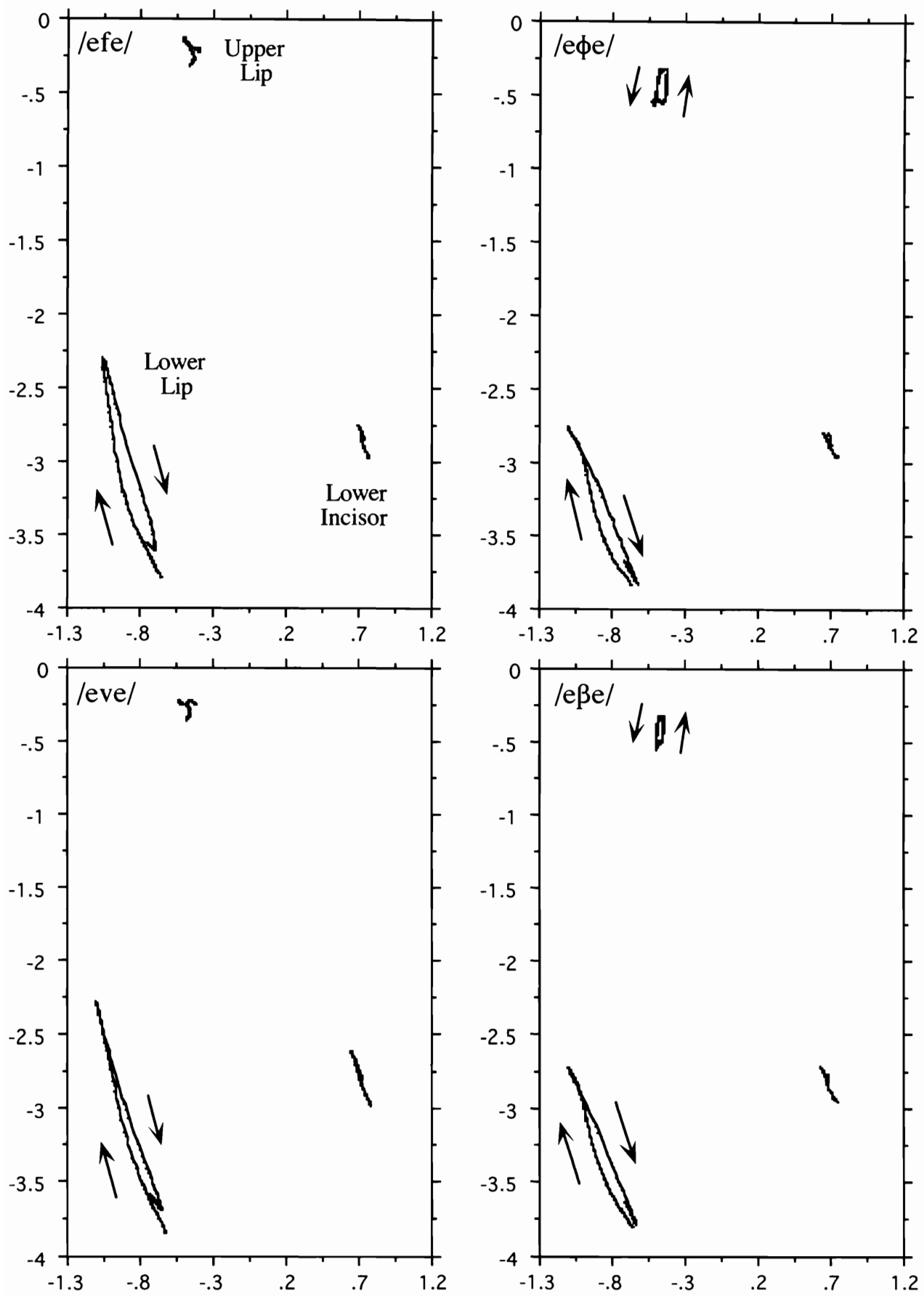

Figure 2. Lip and jaw movement trajectories for Speaker M. 
A major difference between the two speakers concerns the angle of the lower lip movements, which are upwards and backwards for Speaker V, but upwards and forwards for Speaker M. The upper lip also moves backwards for Speaker V and forwards for Speaker M in bilabials. Both lips display a backwards looping motion for the bilabials in Speaker V's production, unlike the more linear movement seen for the labio-dentals. There is no parallel difference between bilabials and labiodentals for Speaker M.

In the next paragraphs, a more detailed look will be taken at individual aspects of the production of these segments, with statistical analysis where this seems appropriate. The maximum elevation, vertical displacement and horizontal positioning of the lower lip, as well as the movements of the jaw and upper lip will all be examined.

The mean values of the peak vertical height of the lower lip (the maximum value of the lower lip coil in the $\mathrm{Y}$ dimension measured down from the origin of the coordinate system) for the four fricatives are graphed in Figure 3. The mean difference between bilabials and labio-dentals is about $5.7 \mathrm{~mm}$, with Speaker V showing a larger difference $(6.4 \mathrm{~mm})$ than Speaker $\mathrm{M}(4.7 \mathrm{~mm})$. In a three-way analysis of variance with place, voicing, and speaker as main effects, this difference is statistically significant. There is also a significant difference between the speakers (presumably mainly due to individual physiological differences which inter alia affect where the origin of the coordinate system falls) and a significant place/speaker interaction. There is no difference in the lower lip elevation between the voiced and voiceless counterparts.

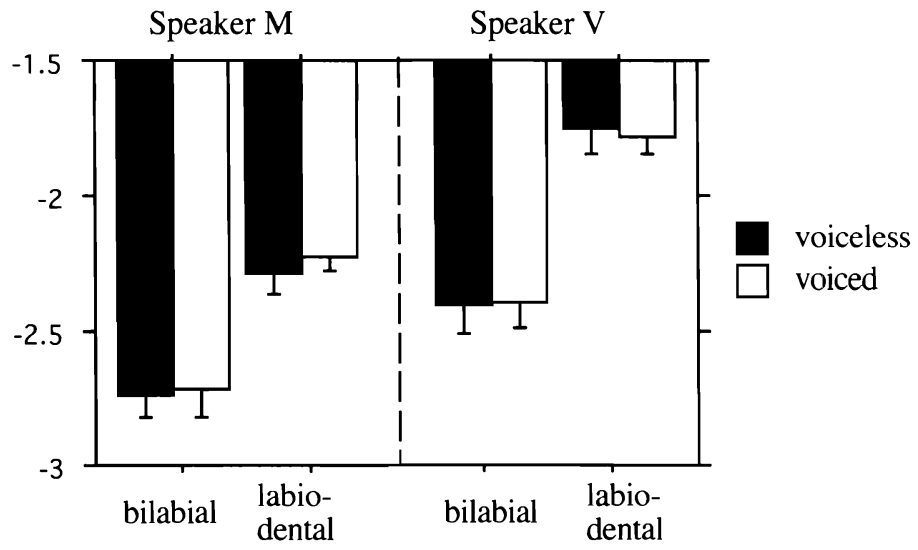

Figure 3. Maximum vertical elevation in cm of the lower lip, by speaker, place and voicing.

Error bars are one standard deviation. 
The total displacement of the lower lip in the vertical dimension, measured as the difference between the height of the receiver coil at the onset of upward movement in the preceding vowel and its maximum elevation, is also significantly greater for the labio-dental $(1.67 \mathrm{~cm})$ than for the bilabial fricatives $(1.14 \mathrm{~cm})$. This is not due to a lower starting position for one than for the other, but entirely to a higher ending position. As shown in figure 4, the difference in displacement is slightly greater for Speaker V than for Speaker M, and Speaker V shows a trend for the voiced fricatives to have greater displacement than their voiceless counterparts. However the difference between the speakers is not statistically significant, and there are no significant interactions between place, speaker and voicing.

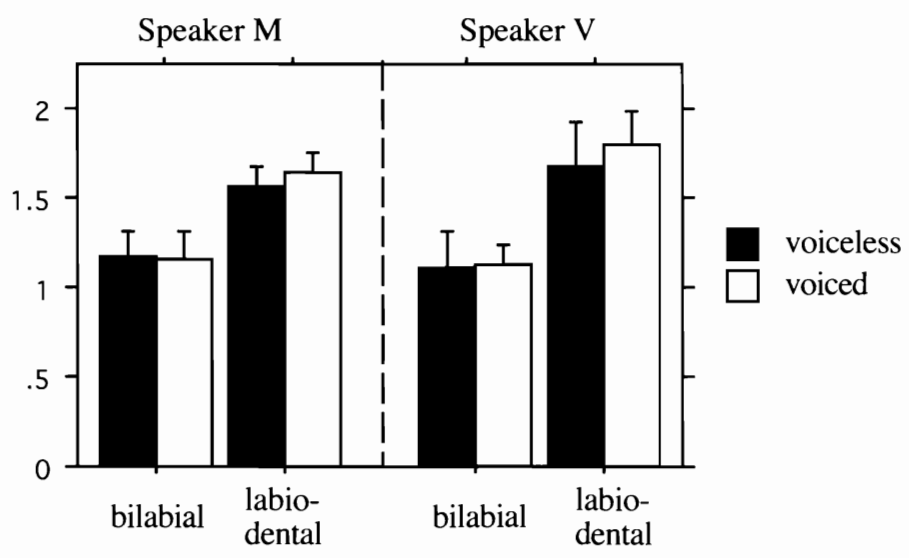

Figure 4. Vertical displacement in $\mathrm{cm}$ of the lower lip, by speaker, place and voicing. Error bars are one standard deviation.

In figure 5, the lower lip vertical displacement is broken down into the component attributable to jaw raising (difference in height of the receiver on the lower incisor) and the residual displacement of the lip itself, usually referred to in the literature as its net displacement. The jaw movement on average only accounts for about $17 \%$ of the total lip movement, hence its contribution is relatively small. However, there is significantly greater jaw displacement for labio-dental than for bilabial fricatives, so jaw movement is assisting in reaching the higher elevation required for labiodental constriction. There is also significantly more jaw displacement for voiced than for voiceless fricatives. The pattern, particularly for Speaker M, suggests a weak trading relationship exists between jaw and lip movement associated with the voicing contrast, with the jaw doing a greater proportion of the work to raise the 
lip in voiced cases than it does in voiceless one. There are small but non-significant negative correlations between vertical jaw displacement and net displacement of the lower lip across each of Speaker M's bilabial and labio-dental sets of tokens considered separately.

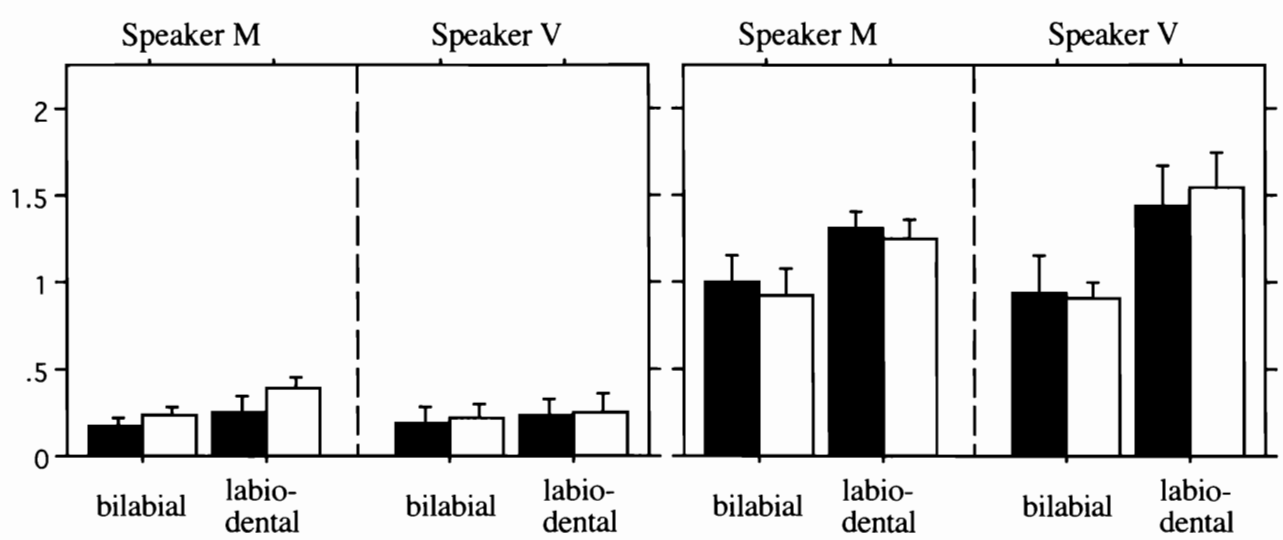

Figure 5. Mean vertical displacement in cm of jaw (left panel) and net displacement of lower lip (right panel), by place, speaker and voicing.

The upper lip shows a noticeable downward movement for bilabials. For Speaker $\mathrm{V}$ the mean downward displacement (the difference between the starting height during the preceding vowel and the lowest position reached during the fricative) is $5.2 \mathrm{~mm}$ in $/ \phi /$ and $3.8 \mathrm{~mm}$ in $/ \beta /$; for Speaker $M$ it is $2.7 \mathrm{~mm}$ in $/ \phi /$ and $2.5 \mathrm{~mm}$ in $/ \beta /$. This movement is apparently arrested by the upward movement of the lower lip, more noticeably so for speaker $\mathrm{M}$ than for speaker $\mathrm{V}$. The upper lip seems to be pushed back up until the lower lip begins to lower, at which point the upper lip resumes its downward movement for a while. This pattern results in a double negative peak in the upper lip movement. Individual tokens vary as to whether the first or second of these points is lower, but it is more often the first for Speaker M and the second for Speaker V.

Averaged vertical movement traces over time for the upper and lower lip coils for the bilabial fricatives produced by Speaker $M$ are shown in figure 6 . Values for 10 repetitions of /eфe/ and for 9 repetitions of /eße/ were aligned at the mid-point of the frication duration $(500 \mathrm{~ms})$ and the mean calculated. This procedure naturally smoothes the curves, and obscures token-to-token variation, but retains any robust features of the pattern. Since the displacement of the upper lip is considerably smaller than that of the lower lip, different scales are used in this 
figure for the upper and lower lip traces, with the magnitude of the upper lip movement (left-hand scale) shown four times greater than that of the lower lip (right-hand scale). Both scales show distances in $\mathrm{cm}$.
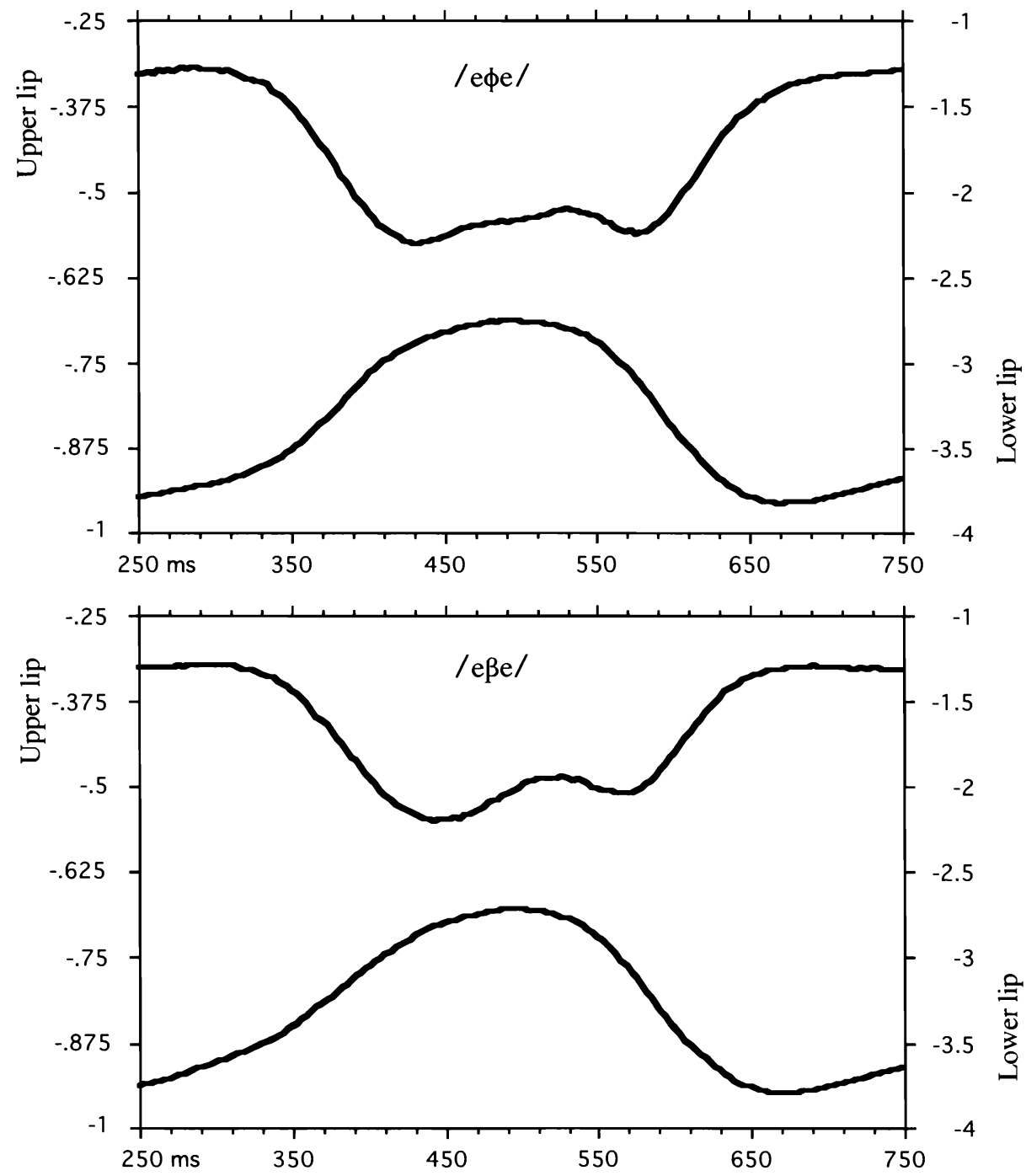

Figure 6. Averaged upper and lower lip vertical movements over time in bilabial fricatives of Speaker $M$. 
Some aspects of the movements shown in these averaged traces are a little puzzling. The upper lip continues to move upwards for a short while after the point of maximum elevation of the lower lip, then lowers a little before finally raising to the position for the vowel. Examination of individual tokens makes clear that this is not an artifact of the averaging procedure, but is the typical pattern. This issue will be revisited in the discussion section of the paper.
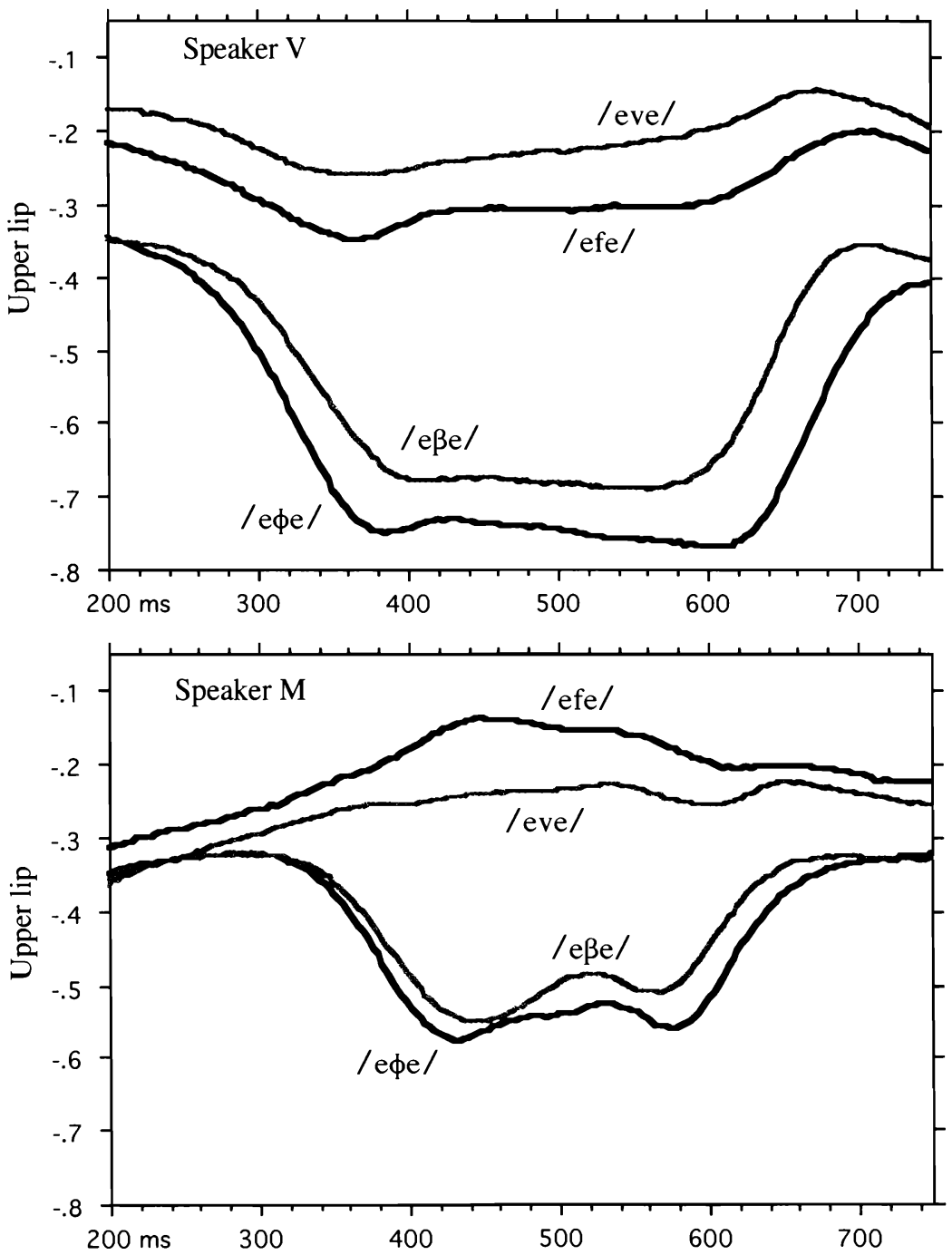

Figure 7. Averaged upper lip vertical movement traces over time for bilabial and labio-dental fricatives, by speaker. 
Upper lip movement in the labio-dental fricatives is of much smaller magnitude than for the bilabials and is inconsistent in direction between the two speakers. The averaged upper lip vertical movement traces for all four fricatives are compared in figure 7 for the two speakers separately. Both speakers have fairly marked downward movements during the bilabial fricatives, which appears to be checked by the upward movement of the lower lip, as discussed above. In the labio-dental fricatives, Speaker V shows small downward movements, and Speaker M small upward movements. In all cases the voiceless member of the fricative pair displays a slightly larger excursion than the voiced member. Figure 7 also demonstrates that the duration of the upper lip's lowering movement in bilabials is clearly shorter in the voiced fricative than in the voiced.

The most extreme location in the horizontal dimension for the lower lip for all the fricatives is shown in Figure 8. Recall that for Speaker V the lower lip movements are backward, whereas for Speaker M the movements are forward, so that this figure shows the most backward position reached by the lower lip for Speaker V, and the most forward position reached by the lower lip for Speaker M. For Speaker $\mathrm{V}$ there is a significant difference between the places in the culminating horizontal position of the lower lip, with the bilabial fricatives having a further forward position. There is a minimal difference in the same direction between the fricatives for Speaker M. Voicing has no significant effect on the horizontal location.

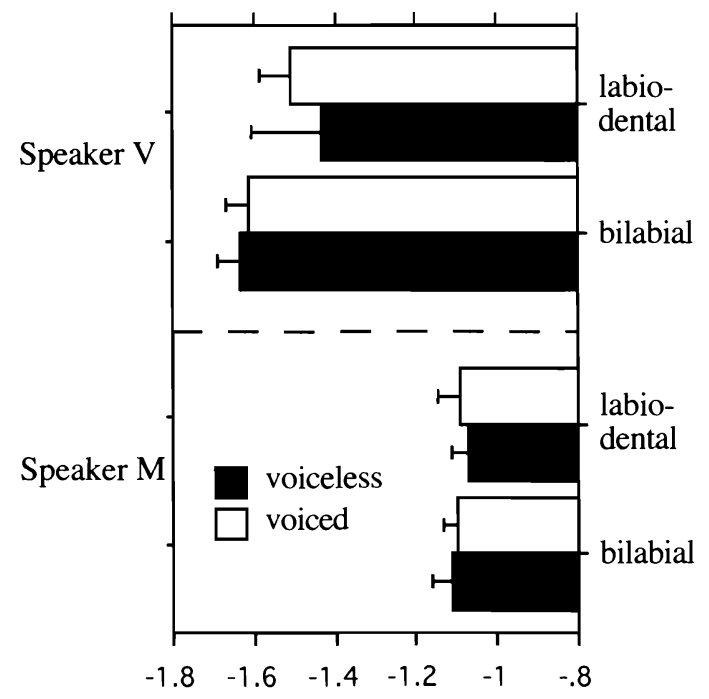

Figure 8. Culminating horizontal position of the lower lip by place, speaker and voicing. 


\section{Discussion}

In the Ewe EMMA data there are, as expected, obvious differences in upper and lower lip movements related to the place of articulation. No differences in movement extent (lower lip height, frontness or displacement) were found related to the voicing distinction between the fricative pairs. This agrees with the trend in studies of bilabial stops, where the articulatory magnitudes for voiced and voiceless counterparts have been found not to show any reliable differences either in nonsense utterances (e.g. Smith 1995, Löfqvist \& Gracco 1997) or in real words from several languages (e.g. Maddieson 1993, Löfqvist 2005). However, there is a difference between the voiced and voiceless fricatives in the duration of the articulation, with the voiced counterparts being shorter than the voiceless. The shorter acoustic frication duration of the voiced members of the pairs is therefore due, or due at least in part, to a shorter duration of the movement creating the fricative constriction, rather than being (solely) due to aerodynamic factors, such as the effect of reduced transglottal air-flow decreasing air-flow past the fricative constriction, and hence reducing the duration of audible frication.

It was suggested above that bilabial fricatives have sufficient contact between the lips that the upper lip is displaced upwards by the lower. This follows the interpretation offered by Löfqvist \& Gracco (1997) of similar movements observed in the production of bilabial stops. These authors further argue that this pattern provides evidence that the target position for the bilabial closure is above the maximal height reached by the lower lip, that is, there is a "virtual target" for the lower lip that is located above the position of the lower lip. This suggestion simplifies the assumptions required concerning the control strategy for achieving closure, since factors like the starting lip configuration can be ignored. The finding that there is a similar kind of lip interaction in the production of Ewe bilabial fricatives casts some doubt on the strength of this argument. Since producing a fricative requires a rather precise degree of constriction short of closure, it seems unlikely that the lower lip target is above the upper lip position, since nothing would impede continuing upward movement of the lower lip to form an inappropriate complete closure. It may therefore be the case that the upward movement of the upper lip during the middle portion of the bilabial fricatives is an active gesture that is necessary to maintain the appropriate lip aperture for the fricative. This hypothesis is also more consistent with the anomaly in timing between the lower and upper lip movements noted in connection with figures 6 and 7 above. If the upper lip is actively raised, then there is no reason to expect this movement to coincide with the maximum elevation of the lower lip. Löfqvist 
(2005) does not include any discussion of lip interaction in fricatives in a paper primarily devoted to examining production of long and short bilabial stops and nasals, but this is probably because there was none to be observed. His data does include tokens of the Japanese loanwords romanized as tofuru ('the TOEFL exam') and daffuru ('duffle') containing the allophone of $/ \mathrm{h} /$ which occurs before the vowel /u/ (romanized as $<u>$ ). This allophone is usually described as a voiceless bilabial fricative, and is so described by Löfqvist, but it is not comparable to the truly fricative segment $/ \phi /$ in Ewe. It is produced by many Japanese speakers as an approximant. For example, Uehara \& Kiyose (1974: 2) note that it "should be pronounced in the same way as the "wh" in "who" with a noticeable spreading of the lips." For three of the four speakers Löfqvist studied there is almost no movement at all for the upper lip in the daffuru tokens. In tofuru two of the speakers show a backward and lowering movement of the upper lip which is almost certainly associated with the unrounding gesture in the transition from $/ \mathrm{o} /$ to /u/ rather than with the articulation of the intervening consonant. In no case is there a lowering then raising of the upper lip of comparable magnitude to that seen in the bilabial fricatives of the Ewe speakers. Nor can such movements be seen in the optically tracked movement traces published by Gomi et al (2002, Figure 4). Of course, it should be remembered that the movements tracked with the EMMA system are those of the receiver coils. In the case of the lips, these are attached at the outer surfaces of the lips near the vermilion border. Because their movements may include "rotational" components of the outer lip surfaces which are to some degree independent of the degree of proximity of the more inward surfaces where labial contact occurs, some caution must always be exercised in interpreting what these movements mean.

As for the suggestion that labial fricatives are subject to phonetic "enhancement", the present data indicate that both proposed types of enhancement are absent. As figure 7 showed, the upper lip movements are minimal in /f/ and /v/, and in conflicting directions for the two speakers. The video recordings of multiple speakers referred to earlier showed no visible distinction between the position of the upper lip during consonants which involve no labial activity such as the velar stops $/ \mathrm{k} /$ and $/ \mathrm{g} /$ and that in the labio-dental fricatives. There is absolutely no evidence that labio-dental fricatives are habitually produced with rounding or protrusion, as suggested by Pulleyblank (2003). The most forward position of the lower lip in labio-dentals is behind that in bilabials for both speakers.

The present data has shown that the articulatory differences between bilabial and labio-dental fricatives involve clearly distinct configurations and control strategies. In bilabials both lips are active, and the upper lip may be playing a 
crucial role in maintaining an appropriate constriction degree. In labio-dentals the upper lip is essentially uninvolved, and the jaw is recruited to a greater degree to assist the lower lip in reaching a more extreme position. From a speaker's point of view, then, the differences between these segments are not especially subtle. It may well be the case that, despite the fact that any difference in the spectrum of the fricative noise between bilabial and labio-dental fricatives is subtle, the complex of cues available from formant transitions (particularly a lower F1 adjoining bilabials) and the visual distinctiveness of the lip configurations make this difference not so difficult for listeners to recognize after all.

\section{REFERENCES}

Dunn, Margaret. 1993. The Phonetics and Phonology of Geminate Consonants: A Production Study. Ph. D. dissertation, Yale University.

Fujimura, Osamu. 1961. "Bilabial stop and nasal consonants: A motion picture study and its acoustical implications." Journal of Speech and Hearing Research 4: 233-46.

Gomi, Hiroaki, Masaaki Honda, Takayuki Ito, and Emi Z. Murano. 2002. "Compensatory articulation during bilabial fricative production by regulating muscle stiffness." Journal of Phonetics 30: 261-79.

Keating, Patricia A. 1984. "Phonetic and phonological representation of stop consonant voicing." Language 60: 286-319.

Ladefoged, Peter. 1964 A Phonetic Study of West African Languages. Cambridge: Cambridge University Press (2nd edition, 1968).

1990. "What do we symbolize? Thoughts prompted by bilabial and labiodental fricatives." Journal of the International Phonetic Association 20(2): 33-36.

Ladefoged, Peter. 1993. A Course in Phonetics, 3rd ed. New York: Harcourt, Brace, Jovanich.

Löfqvist, Anders, and Vincent Gracco. 1997. "Lip and jaw kinematics in bilabial stop consonant production." Journal of Speech-Language-Hearing Research 40: 877-93.

Löfqvist, Anders. 2005. "Lip kinematics in long and short stop and fricative consonants." Journal of the Acoustical Society of America 117: 858-78.

Lubker, James F., and Pamela J. Parris. 1969. "Simultaneous measurements of intraoral pressure, force of labial contact, and labial electromyographic activity during production of the stop consonant cognates $/ \mathrm{p} /$ and $/ \mathrm{b} /$." Journal of the 
Acoustical Society of America 47: 625-33.

Maddieson, Ian. 1984. Patterns of Sounds. Cambridge: Cambridge University Press.

1993. "Investigating Ewe articulations with electromagnetic articulography." Forschungsberichte, Institut für Phonetik und Sprachliche Kommunikation der Universität München 31: 182-214.

1995. "Gestural economy." In Proceedings of the 13th International Congress of Phonetic Sciences (Stockholm), ed by Kjell Elenius and Peter Branderud, vol. 4, pp. 574-77. Stockholm: KTH and Stockholm University. Massaro, Dominic W. 1997. Perceiving Talking Faces: From Speech Perception to a Behavioral Principle (Cognitive Psychology). Cambridge, MA: MIT Press.

Padgett, Jaye, and Marzena Zygis. 2003. "The evolution of sibilants in Polish and Russian." ZAS Papers in Linguistics 32: 155-74.

Perkell, Joseph, Michael Cohen, Mario Svirsky, Melanie Matthies, I. Garabieta, and Michel Jackson. 1992. "Electromagnetic midsagittal articulometer (EMMA) systems for transducing speech articulatory movements." Journal of the Acoustical Society of America 92: 3078-96.

Pulleyblank, Edwin G. 2003. "Non-contrastive features or enhancement by redundant features?" Language and Linguistics 4: 713-55.

Schuh, Russell G. 1995. "Aspects of Avatime phonology." Studies in African Linguistcs 24: 31-67.

Smith, Caroline L. 1995. "Prosodic patterns in the coordination of vowel and consonant gestures." In Papers in Laboratory Phonology: Phonology and Phonetic Evidence, ed. by Bruce Connell and Amalia Arvaniti, pp 205-22. Cambridge: Cambridge University Press.

Stevens, Kenneth N., and S. Jay Keyser. 1989. "Primary features and their enhancement in consonants." Language 65: 81-106.

Sussman, Harvey, Peter F. MacNeilage, and R. J. Hanson. 1973. "Labial and mandibular dynamics during the production of bilabial consonants: preliminary observations." Journal of Speech and Hearing Research 16: 397-420.

Uehara, Toyoaki, and Gisaburo N. Kiyose. 1974. Fundamentals of Japanese. Bloomington, IN: Indiana University Press. 